\title{
THE NAGLER REACTION IN PATHOLOGICAL SERA AND ITS USE AS A LIVER FUNCTION TEST
}

\author{
BY \\ JOHN PERRIN \\ From the Clinical Laboratories, The London Hospital
}

(RECEIVED FOR PUblication MARCh 11, 1959)

The alpha toxin of $C l$. welchii reacts with human serum to produce an opalescence (Nagler, 1939). The nature of the opalescent material is complex, the bulk consisting of lipids and a smaller part of protein (Crook, 1942). This material is derived from lipoprotein complexes, associated with the alpha and beta globulins of the serum, by disruption of their phospholipid components. Purified alpha toxin is a highly specific phosphatase. The action of this phosphatase is directed mainly against lecithin, producing phosphocholine and a diglyceride stearyloleylglyceride (Macfarlane and Knight, 1941). It also acts, but much more slowly, on sphingomyelin to yield phosphocholine and lignoserylsphingosine (Macfarlane, 1942, 1948). The main phospholipid component of human serum is lecithin; the remainder is sphingomyelin and cephalin. These phospholipids are known to be largely metabolized in the liver (Balfour, 1947).

Although the Nagler reaction has been extensively investigated in normal sera, the reaction in sera from pathological states has been neglected. Alterations in phospholipids are common in many diseases, particularly those involving the liver, and might well be expected to influence the result of the Nagler reaction. This paper describes the results of studies of the Nagler reaction in pathological sera, one of the most interesting phenomena being a specific flocculation reaction in parenchymatous liver cell damage.

\section{Materials and Methods}

Nagler Reaction.-The $\mathrm{Cl}$. welchii alpha toxin was supplied as a dried powder by the Wellcome Research Laboratories, each bottle containing 220 international units. For use, $15.3 \mathrm{mg}$. dried toxin was freshly dissolved in $10 \mathrm{ml}$. borate buffer. This gave a concentration of approximately 1.5 international units per $\mathrm{ml}$. A more convenient standard is to use 1 international unit per $\mathrm{ml}$.

Borate Buffer.-Sorenson's at $p \mathrm{H} 7.61\left(20^{\circ}\right): 475$ ml. $0.1 \mathrm{~N} \mathrm{HCl}+525 \mathrm{ml}$. of borate made up of 12.404 g. boric acid $+100 \mathrm{ml}$. $\mathrm{N} \mathrm{NaOH}$ per litre distilled water.

$0.025 \mathrm{M} \mathrm{CaCl}_{2}$.

Sterile serum was used freshly separated at $37^{\circ} \mathrm{C}$. and stored at $+4^{\circ} \mathrm{C}$.

Alpha Toxin Turbidity and Flocculation Tests.Three 3 in. $\times \frac{1}{2}$ in. test-tubes were placed in a waterbath rack. Into two tubes were measured $0.4 \mathrm{ml}$. serum $+0.1 \mathrm{ml}$. $\mathrm{CaCl}_{2}+3 \mathrm{ml}$. borate buffer $+0.5 \mathrm{ml}$. alpha toxin reagent. The time of addition of the toxin was noted and the rack placed in a water-bath at $37^{\circ} \mathrm{C}$. The third tube acted as a control and $0.5 \mathrm{ml}$. borate buffer replaced $0.5 \mathrm{ml}$. toxin, the other reagents being the same. Of the two tubes containing toxin, one was left undisturbed and was inspected at intervals for macroscopic flocculation (see below); the other was used for turbidity measurements at the following times from the start of the test: half hour, hourly from one to 12 hours (or beyond 12 hours when possible), 24 hours.

Optical density was recorded in a Hilger absorptiometer using filters red $\mathrm{O} 2$ and orange $\mathrm{OY} 2$ as for the thymol turbidity. The blank consisted of the control tube, incubated for the same period of time as the test.

Phospholipid in Serum.-Total phospholipid was estimated by the method of Zilversmit and Davis (1950).

Thymol Turbidity and Zinc Turbidity.-The standard technique was employed, using barium sulphate standard (Shank and Hoagland, 1946). When measuring the zinc and thymol turbidities on solutions used in the Nagler reaction $1 \mathrm{ml}$. of toxin-treated or control serum was mixed with $5 \mathrm{ml}$. of zinc or thymol reagent and the resulting turbidities noted after the standard period of 30 minutes. In the toxin-treated sera the alpha toxin-flocculated material was first removed by repeated filtration through No. 1 Whatman paper.

\section{Results}

Two distinct phenomena resulted from the action of $\mathrm{Cl}$. welchii alpha toxin on human sera. The first reaction, common to all types of sera, both normal and from pathological conditions, was the development of a uniform turbidity, as 
originally described by Nagler. The second and distinct reaction was aggregation of the fine particles responsible for this turbidity into coarse floccules. These were visible to the naked eye and sedimented into a fluffy deposit on standing. This second reaction of flocculation was limited almost entirely to sera from cases of hepatocellular damage, and so exhibited a certain specificity. The two reactions are analysed separately.

The Turbidity Reaction.-The degree of turbidity developing in alpha-toxin-treated sera was studied over a period of 24 hours. Observations were usually made hourly in the first half of this period, but were not taken during the night, i.e., 12-24 hours. A final reading was taken at the end of the 24-hour period. The development of the turbidity in all cases followed a smooth hyperbolic curve as might be expected in an enzymic reaction. The 24 -hour readings were used for comparison, as the reaction was virtually complete in the majority of sera by that interval. Total phospholipid estimations (Zilversmit and
Davis, 1950) were done in parallel on each serum and compared with the degree of turbidity. This comparison is shown in Fig. 1 in 150 sera from 10 normal controls and 104 patients suffering from liver disease, namely, infectious hepatitis 12 , serum hepatitis three, glandular fever hepatitis four, subacute hepatitis and cirrhosis of the liver 18 , alcoholic cirrhosis three, obstructive jaundice (extrahepatic) 10, chlorpromazine jaundice two, phosphorus jaundice one, biliary cirrhosis four, jaundice and/or hepatomegaly of doubtful origin two; from heart disease with hepatomegaly seven; and from heart disease without hepatomegaly three, type II nephritis two, diabetes mellitus two, hypercholesterolaemic xanthomatosis three, idiopathic hyperlipaemia two, xanthomatosis one, myxoedema one, thyrotoxicosis one, sarcoidosis one, chylous ascites one, idiopathic steatorrhoea two, lymphosarcoma one, osteomyelitis one, thrombocytopenic purpura one, myelomatosis 17 .

In nearly all sera there is a significant correlation between the total phospholipid concentration

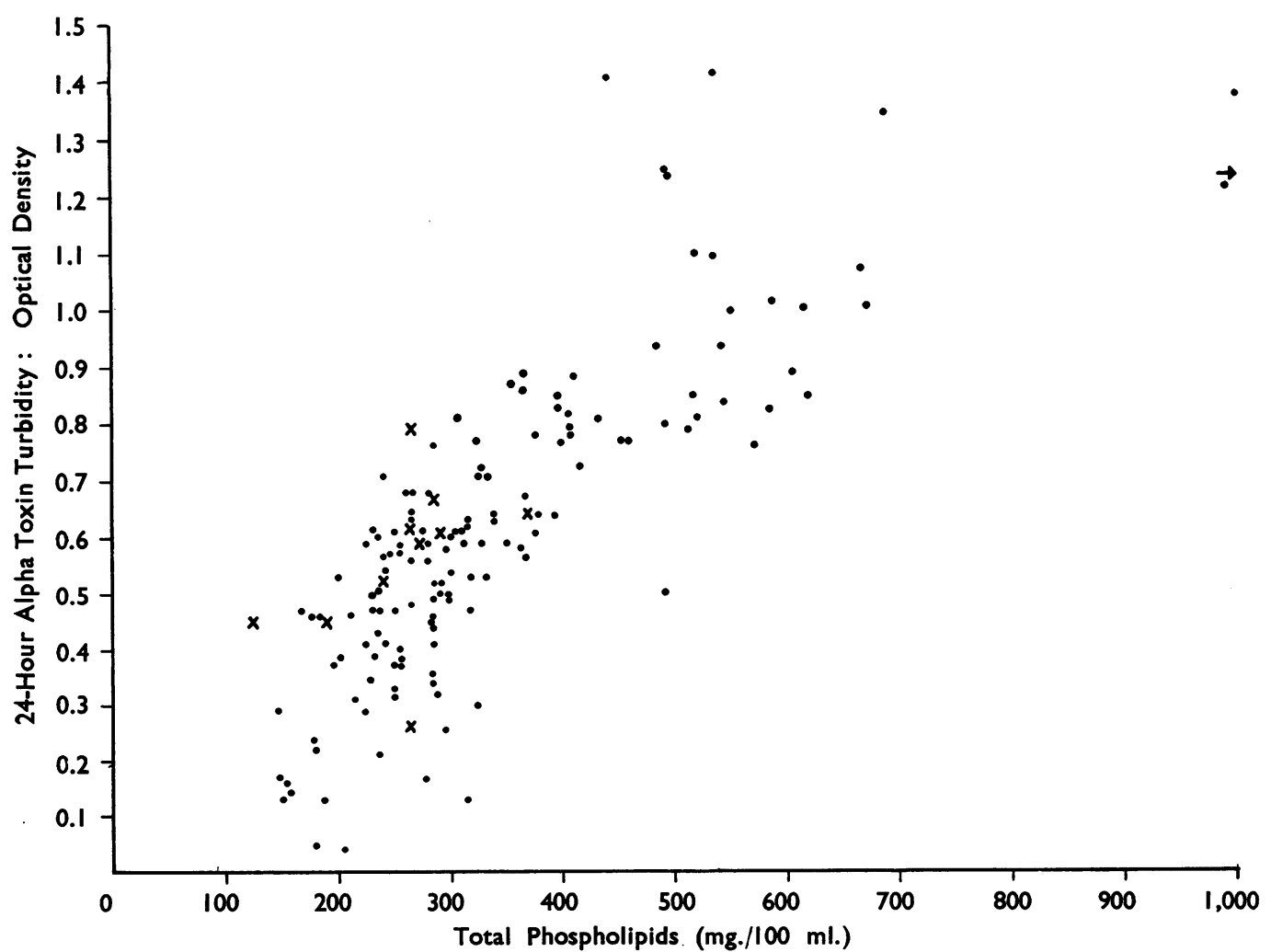

FIG. 1.-The relationship of $\mathrm{Cl}$. welchii 24hour alpha toxin turbidity to total phospholipid content in normal and pathological sera. $X=$ normal sera.

= pathological sera. 
and the degree of turbidity developed in the alpha toxin reaction. Some of the scatter in the results is almost certainly due to phospholipids other than lecithin being included in the total phospholipid figure, but not in the more specific lecithinase reaction. The widest scatter is apparent in sera with very high or very low levels of phospholipid. The group above an optical density of 1.2 are almost entirely derived from cases of xanthomatosis, either hypercholesterolaemia or idiopathic hyperlipaemia. This may represent a significant deviation in these disorders, but too few cases have been studied for a valid analysis. The contrary group of sera with an optical density below 0.25 are nearly all from cases of multiple myelomatosis. Results from 17 cases of multiple myelomatosis are shown separately in Fig. 2 and compared with nine normals. All values for cases of myelomatosis save one lie below the line AB in Fig. 2. Of all human sera examined, both normal and from miscellaneous pathological states, only 12 showed an optical density below 0.25 in the 24-hour toxin turbidity test (Fig. 1). Of these, nine were cases of multiple myelomatosis (approximately $50 \%$ of

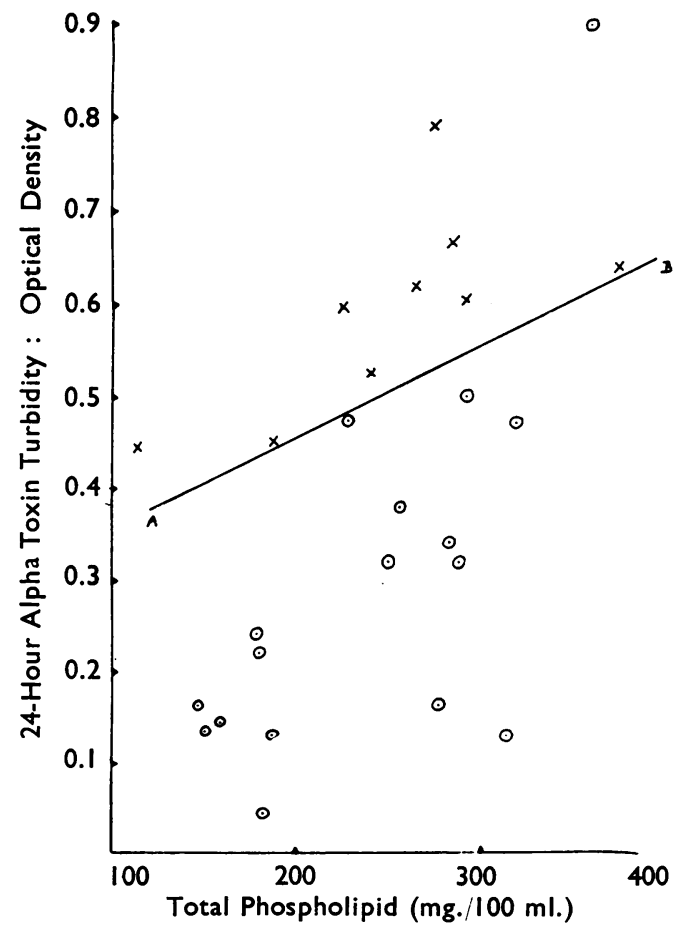

FIG. 2.-The relationship of $\mathrm{Cl}$. welchii 24-hour alpha toxin turbidity to total phospholipid content in nine normal and 17 myelomatosis sera. all cases of multiple myelomatosis). The other three sera showing these exceptionally low figures had liver disease. Two were long-standing and severe cases of cirrhosis, the third a case of transient jaundice, thought to be infectious hepatitis.

As was expected, high toxin turbidity values were found in conditions where lipoproteins are known to be increased. These were any type of obstructive jaundice, extrahepatic or intrahepatic, including the obstructive phase of infectious hepatitis, biliary cirrhosis, idiopathic hyperlipaemia, hypercholesterolaemic xanthomatosis, type II nephritis, and diabetes mellitus. Low values were found in severe cirrhosis of the liver or liver failure, idiopathic steatorrhoea, chylous ascites, and, as already described, in multiple myelomatosis.

The Flocculation Reaction.-While studying the Nagler alpha toxin reaction in sera from pathological conditions, it was noticed that certain of these sera exhibited a coarse, macroscopic flocculation of the liberated particles. This flocculation was entirely independent of the total phospholipid content of the serum, and could occur at high, normal, or low lipid levels. The usual diffuse turbidity reaction always preceded the flocculation in all cases, but the time of flocculation from the initiation of the reaction varied considerably. No flocculation occurred in any of 10 normal sera during a 24hour period of observation.

This flocculation reaction appeared on further analysis to be highly specific for sera from cases of liver disease, characterized by hepatocellular damage. Thus, in 10 out of 11 cases of infectious hepatitis in an active phase following the onset of jaundice, flocculation occurred within a 12-hour period; Case 2 flocculated within 24 hours. Further, the time of flocculation was shortest in the acute phase of the illness and progressively lengthened and recovery ensued (Fig. 3). Similarly flocculation occurred in six sera from three cases of hepatitis in glandular fever, and in four sera from two cases of serum jaundice, all in the period of active cellular damage. Sera from one mild case of glandular fever hepatitis with minimal, transient jaundice, and one case of serum jaundice in the recovery phase, exhibited no flocculation. Flocculation observed at 24 hours represented an unknown time reading between the last recorded reading on the previous evening and the final 24-hour morning reading. This interval is represented by the shaded areas in Fig. 3. Thus in the final stages of recovery of Cases 1 and 2 
flocculation occurred at an unknown time between 12 and 24 hours.

Out of 21 cases of subacute hepatitis or cirrhosis, 12 sera from 11 cases gave a positive flocculation reaction before 12 hours and two sera from two further cases flocculated between 12 and 24 hours. The aetiology of the majority of these cases was obscure, as is usual in developed cirrhosis, but three were preceded by a long alcoholic history, one was associated with a granuloma of doubtful aetiology, possibly sarcoid, and one with extensive secondary carcinomatosis and necrosis. Sera from all 13 cases giving a positive alpha toxin flocculation reaction had raised thymol and zinc turbidity values. In the remaining sera from eight other cases no alpha toxin flocculation occurred. Of these eight negative cases, four had normal thymol turbidity and zinc sulphate turbidity reactions, but the other four had raised values.

As opposed to disease in which hepatocellular damage predominates, in obstructive jaundice of varying aetiology, no flocculation occurred in any sera, although total phospholipid content was usually increased as already indicated. Thus no flocculation was observed in 10 cases of extrahepatic obstruction due to gall-stones and carcinoma, two cases of intrahepatic obstruction due to chlorpromazine, one due to phosphorus poisoning, one case of congenital atresia of the bile ducts with biliary cirrhosis, and three out of four adult cases of biliary cirrhosis in the obstructive phase. One case of biliary cirrhosis did flocculate at 13 hours. The significance of this is discussed later.

In seven cases of liver disease, with palpable enlargement due to chronic heart failure, two gave a positive flocculation reaction only in the 12-24hour period, one at 13 hours, the other at 24 hours. Both cases had normal thymol and zinc turbidity reactions.

Two other cases of liver disease, one jaundiced and one with a palpable liver but not jaundiced, were included in this series, although of doubtful aetiology. Neither showed alpha toxin flocculation.

As controls, in addition to sera from cases of obstructive jaundice, a number of sera from 10 normal people, male and female, 17 cases of myelomatosis, and 22 miscellaneous pathological conditions, as listed in the description of the turbidity reaction, were studied for the flocculation reaction.

None of the control series showed any $ᄋ 007$ flocculation in the first 12 hours of the turbidity

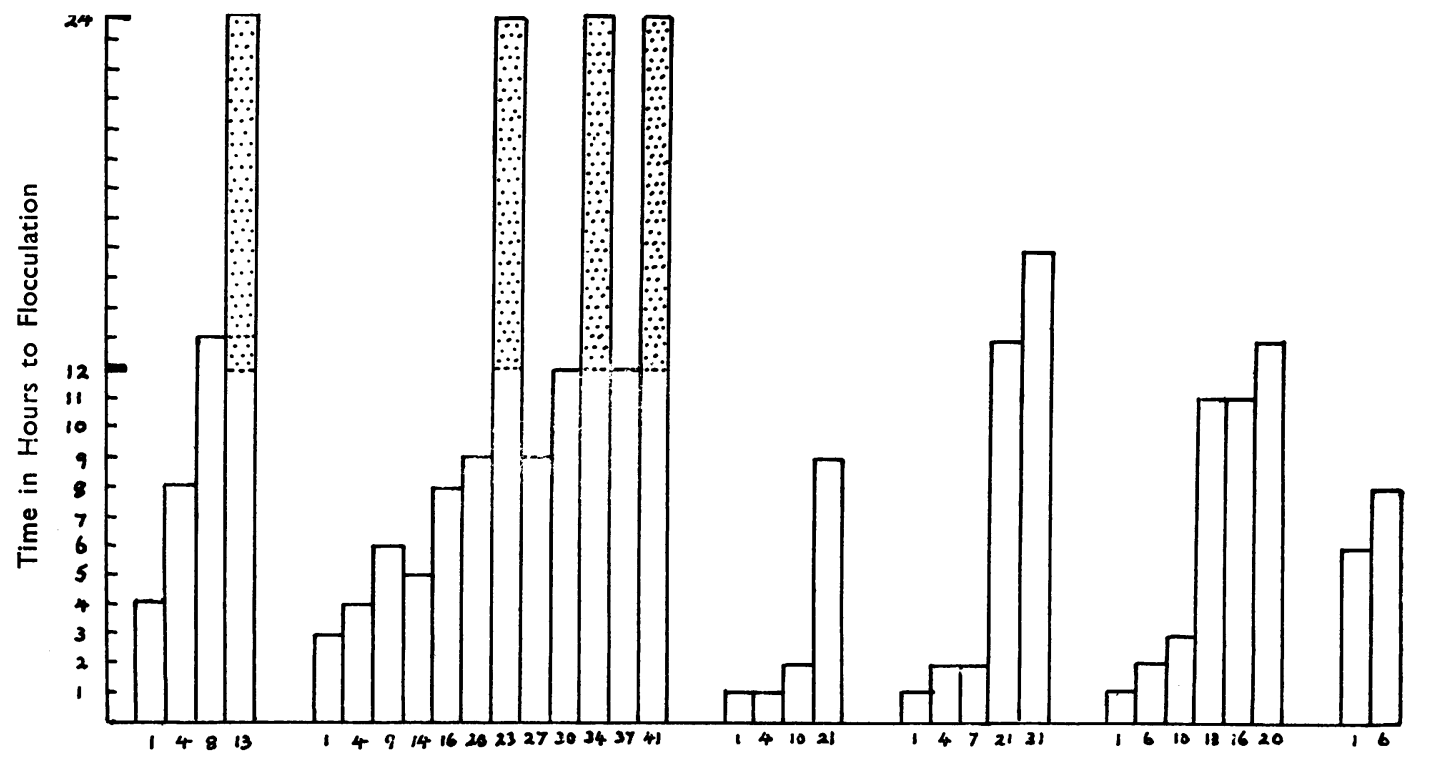

Day of Serum Test after First Serum Examined

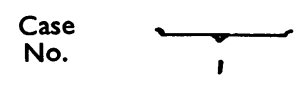

FIG. 3.-Cl. welchii alpha toxin flocculation reaction in six cases of acute infectious hepatitis. 
tests nor did any normal control at the end of 24 hours. Two cases of steatorrhoea, one case of mild heart failure without a palpable liver, and the case of thrombocytopenic purpura with a palpable liver did show flocculation between 12 and 24 hours. In none of these four cases, with the possible exception of the thrombocytopenic patient, could any evidence be found for a pathological lesion of the liver. The thrombocytopenic patient proved in the end to have a diffuse lipoid infiltration of the spleen and liver, without lipaemia, of unidentified origin. It would appear that flocculation up to 12 hours is characterized by hepatocellular damage; flocculation between 12 and 24 hours is less specific and may occur in occasional sera from pathological cases without liver disease, but is obviously rare, and did not occur in the 10 normal controls. If all cases showing flocculation in the 12-24-hour period are excluded, then no false positives are found in any control sera, and four cases are excluded from the total of all liver disease cases showing flocculation.

Role of Phospholipid in Thymol and Zinc Sulphate Turbidity Tests of Liver Function.Both the thymol turbidity and the zinc sulphate turbidity tests are dependent on a complex of protein factors. Turbidity is promoted in both by a high gamma globulin concentration, which forms the bulk of the precipitate (Marrack, Johns, and Hoch, 1950) and a low albumin concentration (Maclagan, Martin, and Lunnon, 1952). The zinc sulphate turbidity is more closely correlated with the globulin content of the serum, whereas the thymol turbidity is partly controlled by the lipoprotein concentration (Maclagan and Bunn, 1947 ; Cohen and Thompson, 1947). As alpha toxin disrupts the major lipoprotein constituents of human serum, the effect on these two turbidity tests should be a measure of their dependence on lipoprotein. The thymol and zinc sulphate turbidities were therefore measured on a number of abnormal sera, where these values were raised in association with liver disease, and compared with values measured after the sera had been exposed to toxin. Results are shown in Table I. All thymol turbidity values were significantly lower, in the majority of cases by 30 to $65 \%$ after lipoprotein disruption by the alpha toxin. The zinc turbidity by contrast showed a variable response. In some cases the turbidity was reduced, but never to a comparable degree as in the thymol reaction; in some cases there was little change; while in a number there was a definite increase in the turbidity. In the latter
TABLE I

CHANGES IN THYMOL AND ZINC TURBIDITY VALUES AFTER TREATMENT OF SERA BY $C L$. WELCHII ALPHA TOXIN

RESULTS WITH NAGLER REACTION

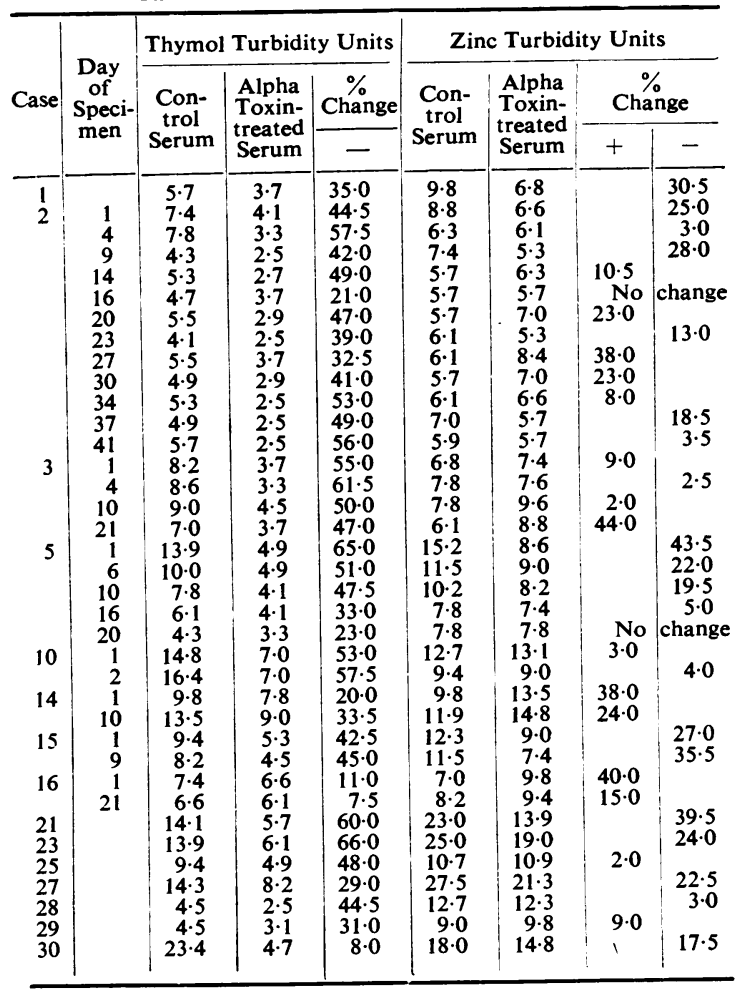

sera destruction of lipoprotein would seem to expose the gamma globulin to the zinc sulphate action with a corresponding increase in metalprotein precipitate.

\section{Discussion}

The phospholipids of human serum exist as complexes with protein, particularly the alpha and beta globulins. In normal serum examined by zone electrophoresis the lipid staining beta component of these lipoproteins is located in the beta globulin region, while the alpha component lies between the albumin and the alpha 1 band (Dangerfield and Smith, 1955 ; Kunkel and Slater, 1952). Distribution and density of radioactive phosphorus incorporated in these complexes closely follow the fat-staining components (Dangerfield and Smith, 1955). The exact mode of linkage of lipid and protein remains obscure. The complex is probably a micelle consisting of a nucleus of phospholipid, the polar groups centrifugally orientated, surrounded by a shell of partly unfolded protein molecules applied to the 
phospholipid. The main phospholipid in human serum is lecithin, accompanied by smaller quantities of sphingomyelin and cephalin. Also centrally integrated in the fat micelle are cholesterol and some neutral fat. The alpha toxin of $\mathrm{Cl}$. welchii disrupts this complex by the action of phosphatase $\mathrm{C}$. Phosphatase $\mathrm{C}$ acts specifically on lecithin at the phosphate bond, to yield phosphocholine and diglyceride such as stearyloleylglyceride (Macfarlane and Knight, 1941). It has a similar but much slower action on sphingomyelin, yielding phosphocholine and lignoserylsphingosine (Macfarlane, 1942, 1948). The whole lipoprotein micelle is consequently fragmented with the liberation of all its component lipids and protein to give the characteristic Nagler turbidity reaction. The released material has been analysed by Crook (1942), and shown to consist of approximately $30 \%$ protein and $70 \%$ fat components, including phospholipid, neutral fat, cholesterol, and fatty acids; small traces of sugar were also present. Petermann (1946) by ultracentrifugation demonstrated the disappearance of the $\mathbf{X}$ component of human plasma following this disruption. The $X$ component is largely composed of beta globulin. The author has confirmed by paper zone electrophoresis that all lipid-staining material is removed from alpha-toxin-treated sera, in both the alpha and beta regions, although the protein pattern is not appreciably altered. The proportion of protein liberated in the Nagler reaction is comparatively small compared with the total serum globulin.

The variables likely to modify the Nagler reaction include total lipoprotein content of serum, the proportion of alpha and beta lipoproteins, and disturbance of liver cell function. The liver is accepted as being the main source of phospholipid synthesis, apart from the intestinal epithelium. Balfour (1947) has suggested, on evidence furnished by studies on the incorporation of radioactive phosphorus in lipoproteins, that all hyperphospholipaemic states, irrespective of the aetiology, are a result of increased synthesis in the liver and not accumulation in the blood stream. Such states include obstructive jaundice, biliary cirrhosis, nephrosis, idiopathic hyperlipaemia, and hypercholesterolaemic xanthomatosis. If confirmed, his conclusion is surprising, since in a condition such as acute virus hepatitis obstruction is accompanied by widespread hepatocellular damage. Individual variations in the alpha and beta components of the lipoproteins in the above conditions have been described by Kunkel and
Slater (1952) and Dangerfield and Smith (1955). In nearly all hyperphospholipaemic sera the beta component was increased. The alpha component was reduced or absent in obstructive jaundice, infective hepatitis in the acute stage, and nephrosis. In diabetes, myxoedema, and xanthomatosis, the alpha component remained. An additional component, the pre-beta lipid, also appeared in certain pathological sera. Ultracentrifuge studies have revealed similar changes. Thus Pierce, Kimmel, and Burns (1954) reported an increase in components $S f$ 0-12, 12-20, and $20-100$ and a decrease in Sf $100-400$ in plasma in acute hepatitis. The study of variations in individual phospholipids has been hampered by the inadequacy of chemical methods available. However, Petersen (1953) found in acute virus hepatitis that the hyperphospholipaemia was due to lecithin components, sphingomyelin and cephalin remaining remarkably constant. In severe liver failure all phospholipids were decreased.

In studying the Nagler reaction, sera from a wide range of different pathological states were sampled, in which lipoproteins might be expected to vary from normal, either in quantity, quality, or relative proportions, and particularly as a result of altered liver metabolism.

The degree of turbidity in the alpha toxin turbidity reaction was roughly proportional to the total phospholipid content of sera (Fig. 1). This correlation held for both normal sera and those from a wide range of pathological conditions. The alpha toxin turbidity presumably parallels lecithin content rather than total phospholipid content. This must account for some of the scatter of the results shown in Fig. 1. Cephalin will contribute to the total phospholipid result but probably not to the Nagler reaction. An increased toxin turbidity ran parallel with increased phospholipids, particularly in obstructive jaundice, biliary cirrhosis, nephrosis, diabetes, idiopathic hyperlipaemia, and hypercholesterolaemic xanthomatosis. The decrease in alpha toxin turbidity in multiple myelomatosis was sometimes, but not necessarily, accompanied by a parallel decrease in the total phospholipid value. Although not exclusive to multiple myelomatosis, such a striking decrease was of diagnostic value. Low values occasionally occurred in liver failure. One possible explanation for the poor reaction in myeloma serum is the presence of a shell of abnormal proteins preventing the penetration of alpha toxin to the nucleus of lecithin in the fat micelles. 
The result of greatest interest was the specific flocculation reaction encountered in liver disease accompanied by parenchymatous cellular damage. Flocculation was entirely independent of the absolute phospholipid content, measured by either alpha toxin turbidity or total phosphorus. Flocculation within 12 hours of the start of the reaction was considered significant, and between 12 and 24 hours less specific, although not occurring in normal sera. Flocculation was constant in all cases of acute virus hepatitis, serum jaundice, and glandular fever hepatitis in the active stages of the disease. The time taken to flocculate increased progressively with recovery (Fig. 3). In chronic hepatitis and cirrhosis of the liver, over $50 \%$ of the cases gave positive alpha toxin flocculation tests. In all these cases the lipidprotein complex released by alpha toxin appeared to be altered in such a way that aggregation of the normally fine particles was encouraged. The obvious explanation for this aggregation was an alteration in the relative proportions of plasma proteins in the surrounding medium, particularly the decrease in albumin and the increase in globulin in liver disease. Flocculation was, however, most constant in acute hepatitis where such an alteration was minimal, and least constant in cirrhosis where the alteration was maximal. Alternatively the liver cell may have a specific metabolic function in linking lipid to protein in the plasma lipoprotein micelle, and this function may be disturbed in extensive liver parenchymatous cell damage. A third possibility is that alteration in the proportion of individual lipoproteins, particularly alpha and beta globulins, may result in predominance of a more flocculable moiety. Changes in alpha and beta lipoproteins in liver disease have already been discussed.' An attempt to measure the proportion of saturated to unsaturated fatty acids in lecithin in liver disease was unsuccessful. Long and Maguire (1954) have shown that phospholipase $\mathrm{C}$ acts only on lecithins containing unsaturated acids in the $C$ position.

Used as a test of liver function, alpha toxin flocculation only approximately correlated with other turbidity tests of hepatocellular disease. In acute infectious hepatitis, glandular fever hepatitis, and serum hepatitis, correlation was good, the alpha toxin flocculation being positive in the acute phase of hepatocellular damage as judged clinically, and by the thymol turbidity and zinc turbidity tests. With recovery, time to flocculation lengthened progressively to beyond the 24hour limit. Alpha toxin flocculation returned to normal before thymol turbidity and zinc sulphate turbidity. In cirrhosis and chronic hepatitis correlation was less good. In all cases where alpha toxin flocculation was positive, thymol and zinc turbidities were increased. But in the eight cases where alpha toxin flocculation was negative four had raised thymol and zinc turbidity values. Such a dissociation presumably reflected the different underlying mechanisms of the various flocculation reactions.

Both thymol and zinc turbidity reactions are dependent on a balance between the various plasma proteins. Gamma globulin promotes and albumin inhibits turbidity (Maclagan et al., 1952). Although the bulk of the precipitate in the thymol reaction is gamma globulin (Marrack et al., 1950), the reaction is also dependent on lipoprotein (Maclagan et al., 1952). The zinc reaction, by contrast, correlates more closely with the gamma globulin content of sera in most pathological states (Kunkel, 1947). The degree of lipoprotein dependence of both tests should be roughly assayable by previous exposure of sera to alpha toxin, which disrupts all lipoprotein. A consistent and appreciable diminution of the thymol turbidity by values between 30 and $65 \%$ resulted in all the sera examined, including cases of acute hepatitis and cirrhosis of the liver (Table I). The same effect was not apparent in the zinc test. Results after exposure to alpha toxin varied considerably, in some sera actually being increased. The importance of lipoprotein in contributing to the thymol reaction was confirmed. In the zinc reaction its removal had little effect, although in some sera this apparently increased the proportion of metal globulin complex precipitated.

\section{Summary}

The Nagler $C l$. welchii alpha toxin reaction has been studied in 150 sera from 10 normal adults and 102 pathological conditions.

The turbidity developed was approximately proportional to the total lipoprotein in all sera.

The majority of sera from 17 cases of multiple myelomatosis gave a poor reaction which, with an abnormally low turbidity, is useful in differential diagnosis.

In hepatocellular damage a specific flocculation occurred within 12 hours of the start of the reaction which was independent of the total lipoprotein content of the sera.

This flocculation characterized all cases of acute hepatitis, including acute infectious hepatitis, serum jaundice, and glandular fever hepatitis. 
Flocculation occurred in over $50 \%$ of cases of cirrhosis of the liver.

Flocculation did not occur in obstructive jaundice of either intra- or extrahepatic type, nor in other types of liver disease.

Destruction of lipoprotein in sera by alpha toxin resulted in a diminution of the thymol turbidity but not of the zinc turbidity reaction.

The author is indebted to the medical and technical staff of the clinical laboratories for all their help. In particular Mr. C. Sait took great trouble in the collection of the necessary specimens.

\section{REFERENCES}

Balfour, W. M. (1947). Gastroenterology, 9, 686.

Cohen, P. C., and Thompson, F. (1947). J. Lab. clin. Med., 32, 314.

Crook, E. M. (1942). Brit.J. exp. Path., 23, 37.

Dangerfield, W. G., and Smith, E. B. (1955). J. clin. Path., 8, 132.

Kunkel, H. G. (1947). Proc. Soc. exp. Biol. (N.Y.), 66, 217.

Kunkel, H. G. (1947). Proc. Soc. exp. Biol. (N.Y.),

Long, C., and Maguire, M. F. (1954). Biochem. J., 57, 223.

Macfarlane, M. G. (1942). Ibid., 36, iii.

(1948) Ibid. 42,587.

587.

and Knight, B. C. J. G. (1941). Ibid., 35, 884.

Maclagan, N. F. and Bunn, D. (1947). Ibid., 41, 580.

Martin, N. H., and Lunnon, J. B. (1952). J. clin. Path., 5, 1.

Marrack, J. R., Johns, R. G. S., and Hoch, H. (1950). Brit. J. exp. Path., 31, 36.

Nagler, F. P. O. (1939). Ibid., $20,473$.

Petermann, M. L. (1946). J.biol. Chem., 162, 37.

Petersen, V. P. (1953). Acta med. scand., 144, 333

Pierce, F. T., Kimmel, J. R., and Burns, T. W. (1954). Metabolism, ierce, F. T.

Shank, R. E., and Hoagland, C. L. (1946). J. biol. Chem., 162, 133

Zilversmit, D. B., and Davis, A. K. (1950). J. Lab. clin. Med., 35, 155. 